\title{
Impact of the Ga Droplet Wetting, Morphology, and Pinholes on the Orientation of GaAs Nanowires
}

\author{
Federico Matteini, $^{\dagger}$ Gözde Tütüncüoglu, ${ }^{\dagger}$ Dmitry Mikulik, $^{\dagger}$ Jelena Vukajlovic-Plestina, $^{\dagger}$ Heidi Potts, $^{\dagger}$ \\ Jean-Baptiste Leran, ${ }^{\dagger}$ W. Craig Carter, ${ }^{\S}, \#$ and Anna Fontcuberta i Morral ${ }^{*}{ }^{\dagger}$ \\ ${ }^{\dagger}$ Laboratoire des Matériaux Semiconducteurs, " Institut des Matériaux, École Polytechnique Fédérale de Lausanne, 1015 Lausanne, \\ Switzerland \\ ${ }^{\S}$ Department of Materials Science and Engineering, Massachusetts Institute of Technology, Cambridge, Massachusetts 02139, United \\ States
}

Supporting Information

\begin{abstract}
Ga-catalyzed growth of GaAs nanowires on $\mathrm{Si}$ is a candidate process for achieving seamless III/V integration on IV. In this framework, the nature of silicon's surface oxide is known to have a strong influence on nanowire growth and orientation and therefore important for GaAs nanowire technologies. We show that the chemistry and morphology of the silicon oxide film controls liquid Ga nucleation position and shape; these determine GaAs nanowire growth morphology. We calculate the energies of formation of $\mathrm{Ga}$ droplets as a function of their volume and the oxide composition in several nucleation configurations. The lowest energy Ga droplet shapes are then correlated to the orientation of nanowires with respect to the substrate. This work provides the understanding and the tools to control nanowire morphology in self-assembly and pattern growth.
\end{abstract}

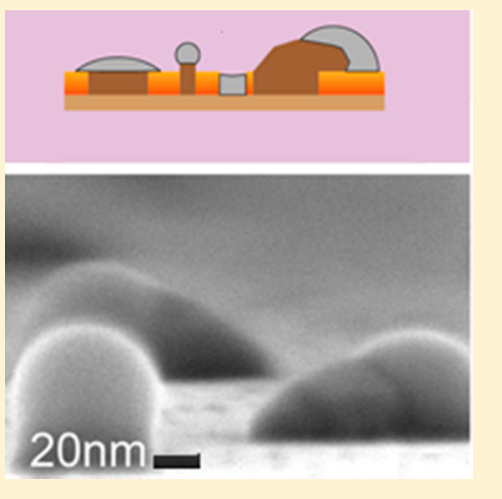

I $\mathrm{n}$ addition to InP, GaAs is well-known to be among the most 1 promising and best performing semiconductor materials for photovoltaic applications because it results in a direct bandgap. ${ }^{1,2}$ However, the high material cost has limited commercial applications. Direct integration of GaAs nanowires on $\mathrm{Si}$ is a potential solution to reduce material consumption and increase cost competitiveness. ${ }^{3}$ To achieve a reliable and high-yield production method, several hurdles exist. Among the different techniques used to produce nanowires, the vapor liquid solid (VLS) technique is the most established: it involves a liquid phase (the catalyst), a solid phase, and a vapor phase. We choose self-catalyzed (i.e., Ga catalyzed) growth to avoid contamination of a foreign catalyst into the nanowire and consequent defect formation of deep trap impurities. ${ }^{4-7}$ Several groups have studied the adoption of the self-catalyzed or catalyst-free growth of GaAs nanowires from GaAs to silicon substrates. $^{8-23}$ Most of these studies focus on steady-state growth and not the initial stages of Ga nucleation on the surface oxide.

Recently, we reported the influence of the native silicon oxide's surface energy on the yield and characteristics of nanowire growth morphology and orientation with respect to the substrate. ${ }^{24}$ In this work, we identify the mechanisms that lead to the different growth orientations. First, we show that the composition of the $\mathrm{SiO}_{x}$ determines the wetting of $\mathrm{Ga}$ droplets as well as their localization via the formation of pinholes.

The $\mathrm{SiO}_{x}$ layers of different thicknesses were obtained by controlled exposure times of bare $\mathrm{Si}$ wafers in a controlled humidity ambient, as reported in ref 24 . In Figure 1, we report the $\mathrm{SiO}_{x}$ (a) wetting properties and (b) chemical composition as a function of the oxide thickness. Figure la shows in green stars the surface energy that would be perceived by a polar liquid at different oxide thicknesses. In this case no trend is observed. However, if we consider the Fowkes model, ${ }^{25}$ the surface energy $\gamma_{i}$ of a substance is broken into independent components: polar, $\gamma_{\mathrm{p}}$, that includes Coulombic forces and any other type of dipole interaction, and dispersive, $\gamma_{\mathrm{d}}$, that derives from van der Waals forces and any other nondipole-related interactions. To assess the effect of the oxidation state the $\mathrm{Ga}$ / $\mathrm{SiO}_{x}$ interfacial energy, we are only interested in the dispersive component, because $\mathrm{Ga}$ is a nonpolar liquid. Given the three unknown $\left(\gamma_{i}, \gamma_{\mathrm{p}}, \gamma_{\mathrm{d}}\right)$, we performed macroscopic $(\sim \mu \mathrm{L})$ contact angle measurements with polar (water and ethylene glycol) and nonpolar (diiodomethane) liquids. These independent observations are used to calculate $\gamma_{\mathrm{d}}$, which is reported in red squares in Figure 1a. Only the dispersive component, $\gamma_{\mathrm{d}}$, of the surface energy changes appreciably with thickness. $\gamma_{\mathrm{d}}$ decreases with increasing thickness and therefore the contact angle decreases with thickness as well. To confirm that the observed trend in contact angle corresponded to a change in $\gamma_{\mathrm{d}}$ and not to nanostructuring of the $\mathrm{Si}$ surface in islands of oxides we characterized the surface roughness of all the $\mathrm{SiO}_{x}$ layers by atomic force microscopy (AFM). The surface roughness was

Received: June 5, 2016

Revised: August 16, 2016

Published: August 26, 2016 


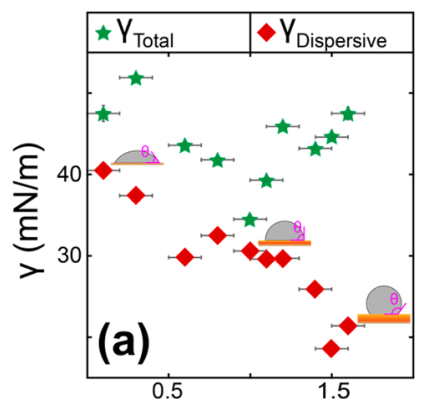

Ox. Thickness $(\mathrm{nm})$

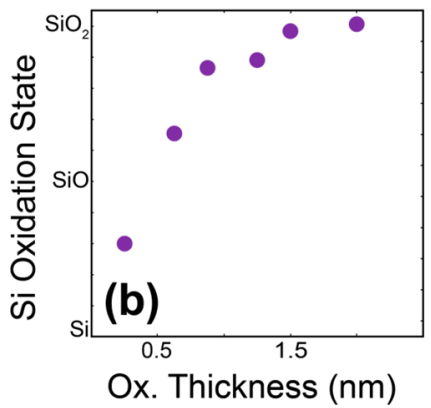

Figure 1. (a) Evolution of the surface energy as a function of native oxide thickness. The dispersive surface energy was calculated with the Fowkes method from the contact angle measurements performed with polar (water and ethilen glycol) and nonpolar (diiodomethane) liquids. (b) Evolution of the silicon oxidation state as a function of the native oxide thickness. The oxidation state of $\mathrm{Si}$ has been calculated with the method described in ref 27 using XPS measurements on different native oxide thicknesses.

very low (RMS $= \pm 0.2 \mathrm{~nm}$ ) for all layers, within AFM tolerance $( \pm 0.2 \mathrm{~nm})$, and no pinholes were observed at this stage. Therefore, the hypotheses of the cause of the change in $\gamma_{\mathrm{d}}$ are that the principle dispersive contribution derives from (i) the $\mathrm{Ga} / \mathrm{Si}$ nonpolar interaction across the $\mathrm{SiO}_{x}$ film, and from (ii) a change in chemical composition of the $\mathrm{SiO}_{x}$ which lead to different surface energies of the film. In case (i) the interaction energy derives from a $1 / r^{6}$ body-body integration across the film thickness. ${ }^{26}$ To verify the contribution of (ii) in the change of contact angle we measured the $\mathrm{SiO}_{x}$ oxygen composition by $\mathrm{X}$-ray photoelectron spectroscopy (XPS) on the different films. The results are shown in Figure $1 \mathrm{~b}$ as a function of $\mathrm{SiO}_{x}$ film thickness. The Si oxygen content has been calculated with the method presented in ref 27 (the Si $2 s, 2 p$ and O XPS data and interpretation are available in the SI). The oxidation state of silicon increases with the oxide thickness and becomes stoichiometric $\mathrm{SiO}_{2}$ beyond 1.4-1.5 nm thickness. These results are consistent with the literature. ${ }^{24}$ In summary, (1) we observe that increasing oxide thickness the chemical composition of the oxide changes toward $\mathrm{SiO}_{2}$, the thermodynamically more stable form of oxidized silicon; (2) the change in chemical composition correlates with decreased wetting (i.e., increased contact angle) of Ga droplets on the $\mathrm{SiO}_{x}$ film.

Since the first publications on Ga-assisted growth of GaAs nanowires, it has been recognized that pinholes have a central role in nucleating and fixing the droplets on the substrate. ${ }^{28}$ Furthermore, pinholes that protrude to the underlying $\mathrm{Si}$ substrate produce an epitaxial relation between the nanowire and the substrate layer during the growth process. ${ }^{28,29}$ However, the pinhole formation mechanism is uncertain. To understand whether or not pinholes are formed in the oxide and if $\mathrm{Ga}$ plays a causal role in pinhole formation, we investigated the surface of thermally treated $\mathrm{Si}$ wafers after the thermal cycling used for growth (same procedure presented in ref 24). We used a Si wafer with a $1.5 \mathrm{~nm}$ oxide. Figure $2 \mathrm{a}$

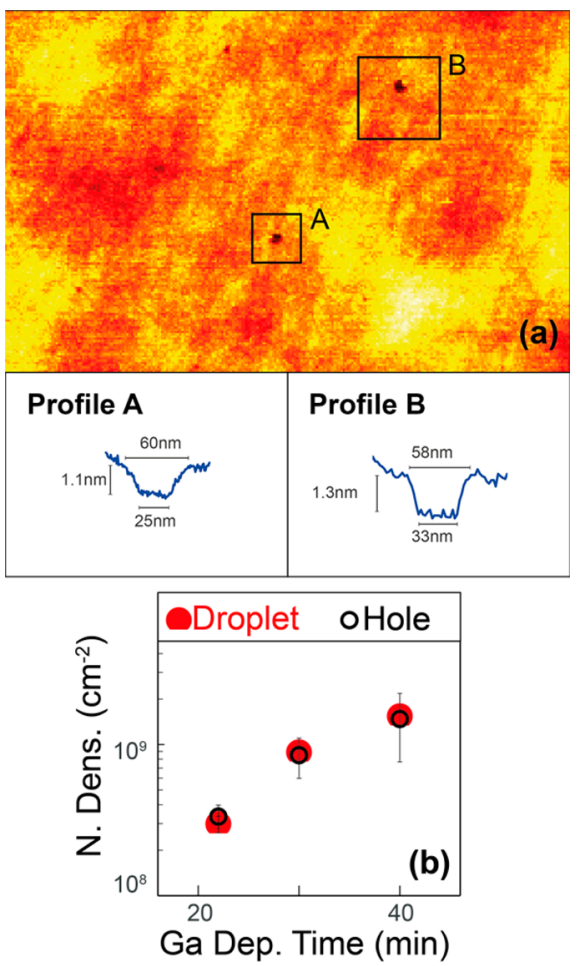

Figure 2. (a) AFM scan of a $1.5 \mathrm{~nm}$ native oxide on $\mathrm{Si}$ (111) substrates after thermal annealing at $750{ }^{\circ} \mathrm{C}$ for $45 \mathrm{~min}$. Insets A and B show the profile of holes in the oxide that were not observed before thermal annealing. (b) Area density of holes and of Ga droplets at different deposition times. The oxide thickness was $1.5 \mathrm{~nm}$. The hole density and diameters were measured by AFM after etching Ga with $25 \mathrm{~min} \mathrm{HCl}$ etch. The plot shows that the density correlate perfectly and that holes are formed during the process.

shows the AFM characterization performed right after the thermal cycle: pinholes of sizes of $\sim 30 \mathrm{~nm}$ are observed in the oxide layer. Insets A and B in Figure 2a show the hole profiles. The depth is comparable to the oxide thickness. Because we did not observe any surface nanostructuring before the thermal cycling, we conclude that pinholes form prior to the deposition, Ga is not necessary for pinhole formation (as already reported $^{30-33}$ ).

We turn now to the role of pinholes in the nanowire growth. We would like to understand if both nanowires and polycrystalline growth, which we will call parasitic growth, start from a pinhole, or whether only nanowires start from pinholes and parasitic growth nucleates on the oxide. With this purpose, we repeated the thermal cycling prior to growth with the addition of $\mathrm{Ga}$ deposition for different time periods, comparing the density of Ga droplets and the density of pinholes. The droplet density was measured by scanning electron microscopy (SEM), and the pinhole density has been measured via AFM after removing the Ga droplets with chemical etching ( $\mathrm{HCl} 37 \% \mathrm{vol}, 25 \mathrm{~min})$. The results are reported in Figure $2 \mathrm{~b}$ : the density of droplets and pinholes is plotted as a function of different Ga deposition times: these densities are perfectly correlated. This observation leads to the 
conclusion that oxide pinholes are the nucleation points for Ga droplets. The selectivity of $\mathrm{Ga}$ deposition on bare $\mathrm{Si}$ agrees with previous reports. ${ }^{34,35}$ Both pinhole and droplet density increase with deposition time. Because the deposition is performed at high temperature, additional pinholes are formed during the process. Further information on the characterization of pinholes and their formation process can be found in the Supporting Information.

To summarize, the $\mathrm{SiO}_{x}$ film's oxygen content increases with film thickness and saturates at $x=2$ around $1.5 \mathrm{~nm}$. Ga becomes increasingly nonwetting as the $\mathrm{SiO}_{x}$ film's thickness increases. $\mathrm{Ga}$ is not necessary for pinhole formation because pinholes form during thermal treatment in UHV. These surface film observations are used to model nucleation and growth of nanowires. The experimental observation of the evolution of the shape of small liquid Ga clusters in nanometer-sized holes is extremely challenging. Here we use a numerical approach to compute the three-dimensional wetting configurations. We assume that the liquid droplets adopt their energy-minimizing shape at any given volume, and that there is no influence of the geometry of the pinhole to the flux of the adatoms due to the extremely low aspect ratio. ${ }^{36}$ Furthermore, we consider several possible wetting configurations and compute their volumedependent minimal energy. For this purpose we used Surface Evolver, a program for minimizing energy of surfaces. ${ }^{37}$

As a first step in modeling, we considered all different possible catalyst droplet configuration in the pinholes and for the different observed contact angles, ${ }^{24}$ as illustrated in Figure 3. Part (a) represents the case of a droplet sitting inside the center of a hole, (b) a droplet sitting in a corner inside a hole, (c) a droplet wetting the walls of a hole, (d) a "spilled" droplet wetting the inside of a hole and also a spilled on one of the abutting $\mathrm{SiO}_{x}$ surfaces, and (e) a droplet symmetrically spilled onto both $\mathrm{SiO}_{x}$ surfaces abutting the pinhole (the computations were performed in three dimensions; the two-dimensional graphics in Figure 3 are for illustrative purposes). The energy difference between configurations are expected to influence the nucleation and growth morphologies. The $\mathrm{Ga}(\mathrm{l}) / \mathrm{GaAs}(\mathrm{s})$ equilibrium contact angle is observed and reported ${ }^{38,39}$ to be bigger than $116^{\circ}$, whereas the $\mathrm{Ga} / \mathrm{SiO}_{x}(1<x<2)$ equilibrium contact angles are always smaller than $116^{\circ}\left(50^{\circ}<\theta<116^{\circ}\right)$. In other words, when supersaturation is reached and the first layer of $\mathrm{GaAs}$ is formed, the droplets tend to populate the energetically more favorable $\mathrm{Ga}(1) / \mathrm{SiO}_{x}$ interface (if still existing).

In the cases of a droplet sitting inside the center of a hole (a) and sitting in the corner of a hole (b) (see Figure 3) in a supersaturated environment, nucleation is more likely at the more favorable $\mathrm{Ga} / \mathrm{Si}(111)$ interface instead of $\mathrm{Ga} / \mathrm{GaAs}$; if the particle touches both surfaces, a Marangoni force will drive the droplet toward the $\mathrm{Ga} / \mathrm{Si}(111)$ interface. This will lead the droplet to fill the hole, being therefore equivalent to the droplet wetting the walls of a hole (c). The latter will most likely lead to the formation of vertical nanowires (see Figure 3c), because there is no imbalance in the forces that would tend to tilt the droplet with respect to the substrate. This is not the case for a droplet that covers the inside of a hole and spills asymmetrically on the $\mathrm{SiO}_{x}(\mathrm{~d})$, since the formation of a Ga/GaAs interface will result in a net force to the droplet to spill out of the hole (see Figure 3d). The "crawling" of the droplet should then be more significant in the case of low oxide thickness (i.e., $\theta<90$; thickness $<\sim 1 \mathrm{~nm})$, since the difference in contact angles before and after the formation of a GaAs nucleus is higher

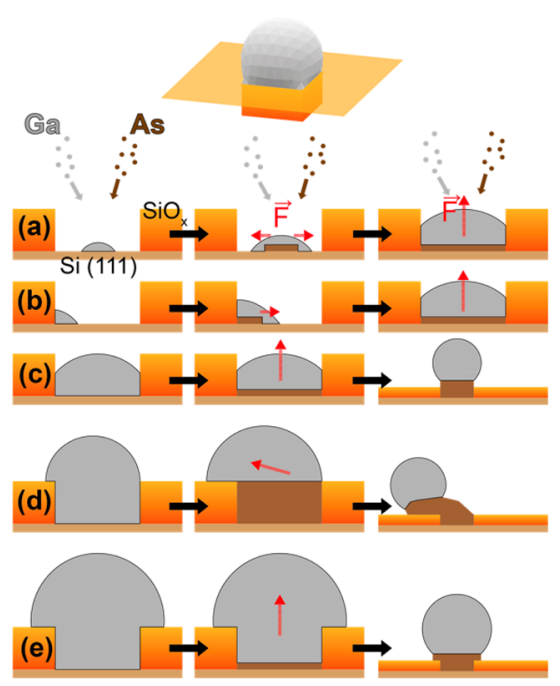

Figure 3. Diagrams of hypothetical droplet configurations prior to nanowire nucleation. The computations presented in this paper were three-dimensional, as shown at the top. The diagrams are used to illustrate the various configurations for simplicity. In orange to yellow, the $\mathrm{SiO}_{x}(1<x<2)$ layer is represented, whereas bare $\mathrm{Si}$ is beige and liquid $\mathrm{Ga}$ is gray and GaAs is brown. The first column presents the droplet configurations before nucleation of the GaAs, the second column the early stages of growth, and the third column the consequent nanowire configuration. In part (a) the droplet is formed in the center of the hole, where the liquid phase forms an interface only with bare Si (111). Part (b) shows the bottom corner droplet configuration, where the liquid forms an interface with bare $\mathrm{Si}$ (111) on the horizontal plane, and with $\mathrm{SiO}_{x}$ on the vertical wall of the hole. In part (c) the droplet is in contact with both sides of the pinhole. Part (d) shows the configuration of an asymmetrically "spilled" droplet which wets only one side of the abutting $\mathrm{SiO}_{x}$ film. In part (e) the case of symmetric spill of the droplet is represented. Its evolution toward a nanowire depends on the contact angle: if $\theta>90^{\circ}$ we observe nanowires (as shown here), whereas $\theta<90^{\circ}$ leads to parasitic growth (not shown).

compared to thicker oxides (i.e., $\theta>90$; thickness $>\sim 1 \mathrm{~nm}$ ). The abrupt change in droplet shape might lead to either the formation of polycrystalline material on the surface or the formation of tilted nanowires.

The homogeneously spilled droplet configuration (e) will behave differently depending on whether the $\mathrm{Ga} / \mathrm{SiO}_{x}$ equilibrium contact angle is larger or smaller than $90^{\circ}$. The case of $\theta>90^{\circ}$ will lead to vertical nanowires, whereas in the case of $\theta<90^{\circ}$, the $\mathrm{Ga} / \mathrm{SiO}_{x}$ interface will be more favorable. In this case the droplet will spill toward the oxide, producing polycrystalline material on the $\mathrm{SiO}_{x}$ or horizontal nanowires. In conclusion, the initial droplet configuration determines the final growth morphologies.

We turn now to the calculation of the probability of finding each proposed configuration. This can be written as

$$
P_{\text {nucl }} \propto e^{-\Delta G / k_{\mathrm{b}} T}
$$

where $P_{\text {nucl }}$ is the nucleation probability of the droplet, $\Delta G$ is the Gibbs free energy, $k_{\mathrm{b}}$ is the Boltzmann constant, and $\mathrm{T}$ is the temperature. The Gibbs free energy can be written as

$$
\Delta G=-\Delta g V+\sum_{i} A_{i} \gamma_{i}
$$

where $\Delta g V$ is the volumetric component and $\sum_{i} A_{i} \gamma_{i}$ is the interface component. The interfacial energy component is 
computed from the minimizing surface, and its value will depend on which surfaces the droplet contacts. In other words, the droplet configurations (a)-(e) will have different nucleation probabilities. Combining the two equations, we can see that the nucleation probability is higher for droplet configurations exhibiting a lower interface energy component for an equivalent droplet volume.

We calculated the energy associated for different droplet configurations at different volumes and for different oxide compositions and thicknesses (i.e., different $\mathrm{Ga} / \mathrm{SiO}_{x}$ equilibrium contact angles $\theta=\left\{59^{\circ}, 76^{\circ}, 82^{\circ}, 94^{\circ}, 98^{\circ}, 106^{\circ}, 116^{\circ}\right\}$, as measured experimentally $\left.{ }^{24}\right)$. Since we did not know the dimensions of the holes at nucleation, we performed the calculation for a range of hole width to height ratio from $2<w /$ $h<20$. For simplicity we report here only the $w / h=2$, the other results, including a detailed discussion of the influence of the aspect ratio of the hole, are available in the Supporting Information. The results are summarized in Figure 4: (a), (b), and (c) show the energy cost as a function of volume for the $\mathrm{Ga} / \mathrm{SiO}_{x}$ equilibrium contact angles $\theta$ of $59^{\circ}, 94^{\circ}$, and $116^{\circ}$. The units of the axes are dimensionless, so that the results can

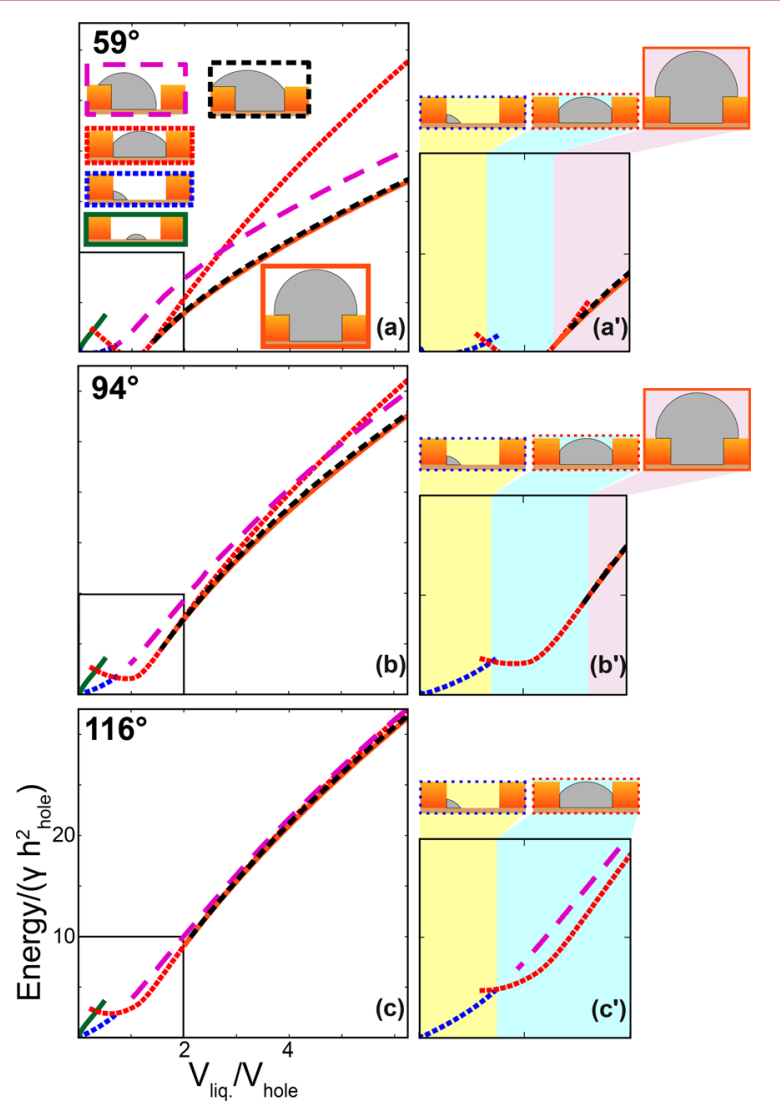

Figure 4. Representation of the energy vs volume curves of the different droplet configurations with (a) $59^{\circ}$, (b) $94^{\circ}$, and (c) $116^{\circ}$ of $\mathrm{Ga}$ to $\mathrm{SiO}_{x}$ contact angles. The green curve represents the droplet at the center of the hole configuration, the blue curve the bottom corner droplet, and the red curve the droplet wetting the walls of the hole. The orange curve is the symmetrically spilled droplet, the black curve is for the nonuniform spilled droplet, and the purple curve is the asymmetrically spilled droplet wetting the bottom of the hole and not one of the pinhole sides. The insets $\left(a^{\prime}\right),\left(b^{\prime}\right)$, and $\left(c^{\prime}\right)$ show a zoomin at small volumes with only the lowest energy cost reported. The background colors refer to the energetically most favorable configuration as a function of the volume, as sketched above (c). be generalized to different material systems. On the $x$-axis we plot the filling percentage of the holes (e.g., when $V_{\text {liq }} / V_{\text {hole }}=1$, the hole is full of liquid), whereas the $y$-axis has the total surface energy normalized by the liquid surface energy multiplied by the squared hole height. Each curve corresponds to a different droplet configuration, as illustrated from the drawings in (a). The $\left(a^{\prime}\right),\left(b^{\prime}\right)$, and $\left(c^{\prime}\right)$ inset show a zoom-in at small volumes, where only the lowest energy configuration curves are reported, with the color background corresponding to the energetically more favorable configuration in each volume range. In (a) we observe that at low volume ratios $\left(0<V_{\text {liq }} / V_{\text {hole }}<0.64\right)$, the lowest energy configuration is the droplet sitting in a corner of the hole, whereas in the range of volume ratios up to 1.28 , the minimal energy droplet wets the walls of the hole and stays pinned to the edges of the hole. Beyond that volume, the droplet will tend to spill symmetrically. However, the nonhomogeneous spill is energetically very close (see Figure $\left.4 a-a^{\prime}\right)$. We expect the statistical differences between the symmetric and nonsymmetric spilled cases to be small.

A similar trend of transition from a droplet sitting in the corner of a hole to wet the walls is also observed in the case of $\theta$ $=94^{\circ}$ and $\theta=116^{\circ}$, but the transitions from the bottom corner to wetting the walls happen at respectively larger volume ratios compared to the $\theta=59^{\circ}$ (for $\theta=94^{\circ}, V_{\text {trans }}=0.7$; whereas for $\theta$ $\left.=116^{\circ}, V_{\text {trans }}=0.74\right)$. For the transition from wetting the walls to spilled, the transition volumes are similarly increased (for $\theta=$ $94^{\circ}, V_{\text {trans }}=1.64$; whereas for $\left.\theta=116^{\circ}, V_{\text {trans }}=2.08\right)$. Consideration of the simulation results of the energy transitions (Figure 4) for the different wetting configurations (Figure 3), we conclude and experimentally observe the following: In the case of $\theta=59^{\circ}$, the $\mathrm{Ga} / \mathrm{SiO}_{x}$ interface is more favorable to form than the $\mathrm{Ga} / \mathrm{GaAs}$, therefore leading either to nanowires or to 2D polycrystalline growth, depending on the volume (i.e., minimizing droplet configuration) at which the nanowire nucleation occurs (see Figure $4 \mathrm{a}$ and Figure 5a). To verify this, we performed a $15 \mathrm{~s}$ GaAs growth on $\mathrm{Si}(111)$ coated with a $\mathrm{SiO}_{x}$ layer of $0.5 \mathrm{~nm}$ (which gives the $59^{\circ} \mathrm{Ga} / \mathrm{SiO}_{x}$ contact angle), for $15 \mathrm{~s}$, in order to be able to observe the early stages. The result is shown in Figure 5d: the growth morphologies attained were vertical nanowires and 2D growth VLS driven, consistent with the prediction. Figure $5 \mathrm{~g}$ shows a growth performed under identical conditions but for $1 \mathrm{~h}$ growth time, to illustrate the evolution of the process.

In the case of $\theta=94^{\circ}$, the energetically favorable configurations (see Figure $4 b-b^{\prime}$ ) are either wetting the pinhole walls or symmetrically spilled (respectively the configuration in Figure $3 c$ and the configuration in Figure $3 \mathrm{e})$, depending on when the solid GaAs nucleation will happen (i.e., Ga droplet volume). For this equilibrium contact angle both configurations, wetting the walls and symmetrically spilled, would lead to vertical nanowire formation, as illustrated in Figure $5 \mathrm{~b}$ - there is no tilting of the droplet with respect to the substrate. However, in the volume range $>1.64$ the nonsymmetric spill of the droplet (configuration in Figure 3d) is energetically close to the homogeneously spilled droplet, potentially leading to a significant amount of $2 \mathrm{D}$ polycrystalline growth. Also in this case we attempted to verify experimentally the prediction by growing GaAs nanowires on a $0.9 \mathrm{~nm}$ oxide layer for $15 \mathrm{~s}$ to observe the early stages of growth (the incubation time had been previously measured to be $\sim 10 \mathrm{~s}^{24}$ ). The results in Figure 5e show vertical nanowires of different lengths and diameter, which may reflect that GaAs nucleation occurred in both the pre- and post-spilling scenarios presented 

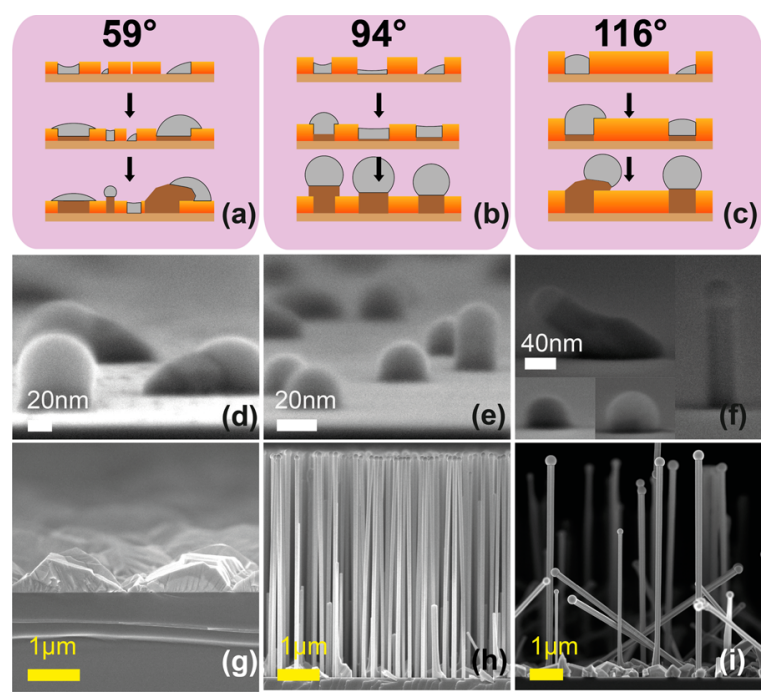

Figure 5. Parts $(a-c)$ illustrate the energetically most favorable droplet configurations and their evolution upon nucleation of the $\mathrm{GaAs}$, for $\mathrm{Ga} / \mathrm{SiO}_{x}$ equilibrium contact angles of $59^{\circ}, 94^{\circ}$, and $116^{\circ}$, respectively. In parts $(\mathrm{d}-\mathrm{f})$ the SEM micrographs of GaAs nanowire grown on $\mathrm{Si}$ (111) coated with $0.5,0.9$, and $1.6 \mathrm{~nm}$ of native oxide are shown. The growth times of $(\mathrm{d}-\mathrm{f})$ correspond to the characteristic incubation time of each oxide thickness, which is $\sim 15 \mathrm{~s}$ for 0.5 and 0.9 $\mathrm{nm}$ oxides, and $\sim 6 \mathrm{~min}$ for the $1.6 \mathrm{~nm}$ oxide (the incubation times were determined in ref 24). Parts $(\mathrm{g}-\mathrm{i})$ show the nanowires grown under the same conditions of $(\mathrm{d}-\mathrm{f})$ after $1 \mathrm{~h}$.

above. Figure $5 \mathrm{~h}$ illustrates the growth after $1 \mathrm{~h}$, yielding to a high-density forest of vertical nanowires.

In the case of $\theta=116^{\circ}$, all the configurations have very similar energies (see Figure 4c), which would not tend to select a particular droplet configuration, and therefore different growth morphologies (2D polycrystalline growth, vertical and tilted nanowires). These potential scenarios are drawn in Figure 5c. The GaAs nanowire growth attempt on oxide thicknesses of $\sim 1.6 \mathrm{~nm}$ (i.e., $\theta=116^{\circ}$ ) for $6 \mathrm{~min}$ (the incubation time had been previously measured to be $\sim 5 \mathrm{~min}^{24}$ ) is shown in Figure 5f. A combination of vertical and tilted nanowires was observed, consistent with the simulation results. Their evolution after $1 \mathrm{~h}$ is shown in Figure 5i.

Our calculations are consistent with the observations that correlate the oxide properties, equilibrium configuration of the $\mathrm{Ga}$ droplets and growth orientation of the nanowires, the combination of the hole dimensions, droplet volume, and surface energy condition with the growth morphology (i.e., vertical nanowire, tilted, or polycrystalline). Nevertheless, since hole formation in the self-assembled growth is distributed over time and so far cannot be controlled, the achievement of only vertical nanowires will depend on controlling the pinhole geometry and evolution.

In conclusion, the native oxide thickness determines the chemical composition of the surface, its thermodynamic stability at high temperature, and its wetting properties. These changes influence the formation of holes in the oxide film, which determine Ga droplet volume and curvature, and the droplet configurations within the pinhole. The latter determines the nanowire nucleation time and the growth morphology. The engineering of the wetting properties of the oxide can control the orientation of the nanowires. These results can be generalized for the case of patterned growth, as preliminary results in the SI point out, and can be extended to different material systems.

\section{ASSOCIATED CONTENT}

\section{S Supporting Information}

The Supporting Information is available free of charge on the ACS Publications website at DOI: 10.1021/acs.cgd.6b00858.

Additional characterization (PDF)

\section{AUTHOR INFORMATION}

\section{Corresponding Author}

*E-mail: anna.fontcuberta-morral@epfl.ch.

\section{Author Contributions}

The manuscript was written through contributions of all authors. All authors have given approval to the final version of the manuscript.

\section{Notes}

The authors declare no competing financial interest.

\section{ACKNOWLEDGMENTS}

The authors thank Stefano Mischler for the XPS analysis and interpretation, Esther Amstad for the insightful discussions and for the preliminary contact angle measurements, and funding through Eranet Rus "Incosin", the Nano Tera project "Synergy", SNF through project no 143908, the ITN Nanoembrace, and the ERC StG UpCon.

\section{REFERENCES}

(1) Shockley, W.; Queisser, H. J. J. Appl. Phys. 1961, 32, 510.

(2) Wallentin, J.; Anttu, N.; Asoli, D.; Huffman, M.; Åberg, I.; Magnusson, M. H.; Siefer, G.; Fuss-Kailuweit, P.; Dimroth, F.; Witzigmann, B.; Xu, H. Q.; Samuelson, 1.; Deppert, K.; Borgström, M. T. Science 2013, 339, 1057.

(3) Krogstrup, P.; Jørgensen, H. I.; Heiss, M.; Demichel, O.; Holm, J. V.; Aagesen, M.; Nygard, J.; Fontcuberta i Morral, A. Nat. Photonics 2013, 7, 306.

(4) Hemesath, E. R.; Schreiber, D. K.; Gulsoy, E. B.; Kisielowski, C. F.; Petford-Long, A. K.; Voorhees, P. W.; Lauhon, L. J. Nano Lett. 2012, 12, 167.

(5) Breuer, S.; Pfüller, C.; Flissikowski, T.; Brandt, O.; Grahn, H. T.; Geelhaar, L.; Riechert, H. Nano Lett. 2011, 11, 1276.

(6) Sze, S. M. Physics of Semiconductor Devices;Wiley-Interscience: New York, 1981.

(7) Slezák, J.; Ondřejček, M.; Chvoj, Z.; Cháb, V.; Conrad, H.; Heun, S.; Schmidt, T.; Ressel, B.; Prince, K. C. Phys. Rev. B: Condens. Matter Mater. Phys. 2000, 61, 16121.

(8) Colombo, C.; Spirkoska, D.; Frimmer, M.; Abstreiter, G.; Fontcuberta i Morral, A. Phys. Rev. B 2008, 77, 155326.

(9) Paek, J. H.; Nishiwaki, T.; Yamaguchi, M.; Sawaki, N. Phys. Status Solidi C 2009, 6, 1436.

(10) Krogstrup, P.; Popovitz-Biro, R.; Johnson, E.; Madsen, M. H.; Nygard, J.; Shtrikman, H. Nano Lett. 2010, 10, 4475.

(11) Mandl, B.; Stangl, J.; Hilner, E.; Zakharov, A. A.; Hillerich, K.; Dey, A. W.; Samuelson, L.; Bauer, G.; Deppert, K.; Mikkelsen, A. Nano Lett. 2010, 10, 4443.

(12) Plissard, S.; Larrieu, G.; Wallart, X.; Caroff, P. Nanotechnology 2011, 22, 275602.

(13) Ambrosini, S.; Fanetti, M.; Grillo, V.; Franciosi, A.; Rubini, S. AIP Adv. 2011, 1, 042142.

(14) Han, N.; Wang, F.; Hou, J. J.; Yip, S.; Lin, H.; Fang, M.; Xiu, F.; Shi, X.; Hung, T.; Ho, J. C. Cryst. Growth Des. 2012, 12, 6243.

(15) Priante, G.; Ambrosini, S.; Dubrovskii, V. G.; Franciosi, A.; Rubini, S. Cryst. Growth Des. 2013, 13, 3976. 
(16) Uccelli, E.; Arbiol, J.; Magen, C.; Krogstrup, P.; Russo-Averchi, E.; Heiss, M.; Mugny, G.; Morier-Genoud, F.; Nygard, J.; Morante, R. J.; Fontcuberta i Morral, A. Nano Lett. 2011, 11, 3827.

(17) Glas, F.; Harmand, J.-C.; Patriarche, G. Phys. Rev. Lett. 2007, 99, 146101.

(18) Ramdani, M. R.; Harmand, J.-C.; Glas, F.; Patriarche, G.; Travers, L. Cryst. Growth Des. 2013, 13, 91.

(19) Dubrovskii, V. G.; Sibirev, N. V. Phys. Rev. B: Condens. Matter Mater. Phys. 2008, 77, 035414.

(20) Krogstrup, P.; Curiotto, S.; Johnson, E.; Aagesen, M.; Nygard, J.; Chatain, D. Phys. Rev. Lett. 2011, 106, 125505.

(21) Krogstrup, P.; Jørgensen, H. I.; Johnson, E.; Madsen, M. H.; Sørensen, C. B.; Fontcuberta i Morral, A.; Aagesen, M.; Nygård, J.; Glas, F. J. Phys. D: Appl. Phys. 2013, 46, 313001.

(22) Kang, J.-H.; Gao, Q.; Joyce, H. J.; Tan, H. H.; Jagadish, C.; Kim, J.; Guo, Y.; Xu, H.; Zou, J.; Fickenscher, M. A.; Smith, L. M.; Jackson, H. E.; Yarrison-Rice, J. M. Cryst. Growth Des. 2011, 11, 3109.

(23) Matteini, F.; Tütüncüoğlu, G.; Rüffer, D.; Alarcón-Lladó, E.; Fontcuberta i Morral, A. J. Cryst. Growth 2014, 404, 246.

(24) Matteini, F.; Tütüncüoglu, G.; Potts, H.; Jabeen, F.; Fontcuberta i Morral, A. Cryst. Growth Des. 2015, 15, 3105.

(25) Fowkes, F. M. Ind. Eng. Chem. 1964, 56, 40.

(26) Argento, C.; Jagota, A.; Carter, W. C. J. Mech. Phys. Solids 1997, $45,1161$.

(27) Himpsel, F. J.; McFeely, F. R.; Taleb-Ibrahimi, A.; Yarmoff, J. A.; Hollinger, G. Phys. Rev. B: Condens. Matter Mater. Phys. 1988, 38, 6084.

(28) Fontcuberta i Morral, A.; Colombo, C.; Abstreiter, G.; Arbiol, J.; Morante, J. C. Appl. Phys. Lett. 2008, 92, 063112.

(29) Mandl, B.; Stangl, J.; Hilner, E.; Zakharov, A. A.; Hillerich, K.; Dey, A. W.; Samuelson, L.; Bauer, G.; Deppert, K.; Mikkelsen, A. Nano Lett. 2010, 10, 4443.

(30) Watanabe, H.; Fujita, K.; Ichikawa, M. Appl. Phys. Lett. 1997, 70, 1095.

(31) Wei, Y.; Wallace, R. M.; Seabaugh, A. C. Appl. Phys. Lett. 1996, 69, 1270.

(32) Xue, K.; Xu, J. B.; Ho, H. P. Nanotechnology 2007, 18, 485709.

(33) Johnson, K. E.; Engel, T. Phys. Rev. Lett. 1992, 69, 339.

(34) Nitta, Y.; Shibata, M.; Fujita, K.; Ichikawa, M. Surf. Sci. 1999, 431,565 .

(35) Shibata, M.; Stoyanov, S. S.; Ichikawa, M. Phys. Rev. B: Condens. Matter Mater. Phys. 1999, 59, 10289.

(36) Vujaklovic-Plestina, J.; Dubrovskii, V. G.; Tutuncuoglu, G.; Potts, H.; Ricca, R.; Meyer, F.; Matteini, F.; Leran, J.; Fontcuberta i Morral, A. Nanotechnology 2016; http://arxiv.org/abs/1608.06156.

(37) Brakke, K. A. Exp. Math. 1992, 1, 141.

(38) Shetty, R.; Balasubramanian, R.; Wilcox, W. R. J. Cryst. Growth 1990, 100, 58

(39) Kashkooli, I. Y.; Munir, Z. A.; Williams, L. J. Mater. Sci. 1974, 9, 538. 